\title{
Residue on Pyriform Sinuses Following Swallowing
}

National Cancer Institute

\section{Source}

National Cancer Institute. Residue on Pyriform Sinuses Following Swallowing. NCI

Thesaurus. Code C127341.

A finding of residue on pyriform sinuses following swallowing. 